\section{§43. Plasma Polarization Spectroscopy}

Fujimoto, T., Iwamae, A. (Dept. Engng. Phys. and Mech., Kyoto Univ.),

Dubau, J. (Paris Observatory),

Goto, M.

When an ensemble of atoms is excited by a beam of electrons, its excitation is anisotropic, and the excited atoms have alignment besides the population. The emitted radiation is linearly polarized. Thus, the polarization characteristics of an emitted line contain information of the anisotropic characteristics of the plasma, namely the anisotropic velocity distribution of electrons. We call this experimental method the Plasma Polarization Spectroscopy (PPS). We first observed polarization of berylliumlike oxygen lines and heliumlike carbon lines from the WT-3 tokamak.

The objectives of this collaboration research were 1 . to investigate various modes of instrumentation of PPS and to optimize the elements of the optical system, and 2. to develop the theoretical framework of PPS so that a quantitative interpretation is possible for observation.

Since we use a calcite plate as a polarization separation element, we did a thorough investigation of the optical characteristics of a calcite plate. The light rays from a virtual light source of a slit go through the plate. Two phenomena appear: 1 . as viewed from the downstream, the horizontal position of the virtual light source shifts in the transverse direction for the extraordinary light component (e-ray) with respect to that for the ordinary light component (o-ray), and 2. the positions of the light source images shift in the direction of the propagation of the light. We constructed a computer code to account for the propagating light rays incident onto a two-dimensional surface of the plate. We calculated these shifts for various wavelengths and for various tilt angles of the plate. For the $\operatorname{HgI} \lambda 253.7$ $\mathrm{nm}$ line we measured the deviation distance of the e-ray image from the o-ray image as a function of the tilt angle of the plate. From the comparison with the calculation we concluded that the optical axis (crystal axis) of our plate is 61 degrees with respect to the surface normal. The similar measurements were done for various wavelengths by using several emission lines from a mercury discharge lamp. The second effect results in a shift of the focal plane of the focused image of the virtual slit. We calculated the shifts for the e-ray and o-ray, and found that, for the $7.5 \mathrm{~mm}$ thick calcite plate, the shift at $\lambda 253.7 \mathrm{~nm}$ is $1.8 \mathrm{~mm}$ longer from the original position for the e-ray and $2.4 \mathrm{~mm}$ for the o-ray. We used a spectrometer with a CCD detector to experimentally verify the calculation. This arrangement is nothing but the mode 1 optical system. We adjusted the position of the CCD detector, and measured the FWHM of the image. From the minimum of the FWHM of the image, we confirmed that our calculation predicts the shifts well.

Since we use a condenser lens in the second mode of the optical system, we investigated the distortion of polarization by a lens. A classical electric dipole emits the $\pi$ polarized light and a concave lens focuses the image of this dipole on the focal plane. Owing to the oblique incidence of the light ray onto the lens surface, the polarization direction may be rotated at the focal position. We calculated this rotation angel for a thick lens. We found that in practical situations the rotation angle does not exceed 1 degree.

We also estimated the magnitude of rotation of the polarization direction when the light goes through a glass window. In normal incidence, no rotation occurs, but with a finite incidence angle polarization direction could be rotated. We found that the rotation angle is less than 2 degrees for incident angles less than 20 degrees.

In the second mode, we use a bundle of optical fibers. These fibers are of the uv grade, and we measured the effective transmission of the fiber for various wavelengths. We found that for longer wavelengths than at $\lambda 400 \mathrm{~nm}$, the transmission is higher than $80 \%$ for the fibers of $25 \mathrm{~m}$ long. However, at $\lambda 253.7 \mathrm{~nm}$ it is $20 \%$ for the $25 \mathrm{~m}$ long fibers and $60 \%$ for the $8 \mathrm{~m}$ fibers. At $\lambda 200 \mathrm{~nm}$ it is virtually 0 for the longer fibers and $30 \%$ for the shorter fibers. We concluded that for measurements of berylliumlike oxygen and heliumlike carbon, the longer fibers cannot be used.

For application of PPS to LHD, we have to use the mode 2 optical system. We tried an achromatic macro lens of a photographic camera in order to avoid the variation of the focal length depending on the wavelength of the light. Unfortunately, the acceptance angle of this lens was too small to obtain good $\mathrm{S} / \mathrm{N}$ ratio signals. We compared the efficiency of focussing the light by a lens and a mirror. We found that the mirror has a higher efficiency by a factor of 5 . We do not understand the reason, but a mirror may be better for the LHD experiment.

We take berylliumlike oxygen for our theoretical analysis. We use cross section values calculated by the distorted wave method in our population-alignment collisional-radiative model. The calculated polarization is about an order smaller than measurement. 